\title{
MicroRNA-135b exerts oncogenic activity in glioblastoma via the inhibition of glycerol kinase 5 expression
}

\author{
YONG YU ${ }^{1}$, JIAN-HUA ZHANG ${ }^{2}$ and BAO-ZHONG ZHANG ${ }^{1}$ \\ Departments of ${ }^{1}$ Neurosurgery and ${ }^{2}$ General Surgery, \\ The Second Artillery General Hospital of The People's Liberation Army, Beijing 100088, P.R. China
}

Received August 29, 2014; Accepted March 23, 2015

DOI: $10.3892 / \mathrm{mmr} .2015 .3708$

\begin{abstract}
Glioblastoma is the most common primary malignancy of the adult central nervous system and is associated with a markedly poor prognosis. Elucidating the pathogenesis and molecular changes will assist in further understanding the pathogenesis and progression of the disease and offer novel targets for therapies. The present study demonstrated that the expression level of GK5 was lower in high-grade glioblastoma tissues compared with low-grade ones and it can promote proliferation in glioblastoma cells. The regulatory mechanism of GK5 in glioblastoma were also investigated. It was revealed that GK5 is a target of miR-135b in U87MG glioblastoma cells. Controry to GK5, the expression of miR-135b is upregulated in glioblastoma and its expression is positively associated with the grade of the disease. Finally, it was demonstrated that miR-135b promoted the proliferation of U87MG cells. Therefore, miR-135b may function as an oncogene by inhibiting GK5 in glioblastoma.
\end{abstract}

\section{Introduction}

Glioblastoma is the most common type of primary malignancy of the adult central nervous system and is associated with a poor patient prognosis. Although radiation therapy (RT) has been shown to prolong overall survival compared with surgery alone (1), patients treated with RT typically experience disease progression within the radiation treatment field. Glioblastomas are infiltrative tumors that typically metastasize to healthy brain tissue.

MicroRNAs (miRs) are small non-coding RNAs that control mRNA stability and the translation of target mRNAs by binding to regulatory sites, which are commonly located in the 3'-untranslated region (3'-UTR) of the transcript (2). It has

Correspondence to: Dr Bao-Zhong Zhang, Department of Neurosurgery, The Second Artillery General Hospital of The People's Liberation Army, 16 Xin Jie Kou Wai Avenue, Beijing 100088, P.R. China

E-mail: 3056175508@qq.com

Key words: microRNA-135b, glycerol kinase 5, glioblastoma recently been reported that miRs are involved in the phenotypic changes in human glioma. For example, miR-21 knockdown inhibits glioma growth in vivo. Furthermore, miR-21 exhibits synergistic cytotoxicity with the neural precursor cell, secreted form of tumor necrosis factor-related apoptosis-inducing ligand (sTRAIL), in human gliomas (3). miR-34a suppresses the development of brain tumor and glioma stem cells (4), miR-181a increases human malignant glioma U87MG cell sensitivity to radiation by targeting Bcl-2 (5). The expression of miR-135b is upregulated in glioma (6) and miR-135b functions as an oncogene in other types of malignant tumors, which suggests that it may be a useful target for developing malignant tumor therapeutic approaches (7-11). However, studies continue to provide evidence for its involvement in glioma. The present study demonstrated that GK5 was a tumor suppressor gene in glioblastoma and that miR-135b acts as an oncogene by regulating the expression of GK5. These findings assist in further understanding the pathogenesis and progression of glioblastoma and offer novel targets for therapy.

\section{Materials and methods}

Immunohistochemistry. Human glioma tissue samples were obtained from The Second Artillery General Hospital of the People's Liberation Army (Beijing, China) and Tongji Hospital (Shanghai, China). In total, 116 samples world health organization (WHO) grade I (benign glioma) and 122 samples WHO grade IV (malignant glioma, also termed glioblastoma) were obtained from 96 females and 142 males with a mean age of 41 years (range, 31-79) (12). Immunohistochemisty was performed using standard techniques. Antigen retrieval was conducted using an autoclave. Incubation was performed using $10 \%$ normal goat serum in phosphate-buffered saline for $15 \mathrm{~min}$ in order to eliminate non-specific staining. Incubation with anti-glycerol kinase 5 (anti-GK5) antibody (cat. no. AB96202; Abcam, Cambridge, MA, USA) was conducted. Sections were counter-stained using 10\% Mayer's hematoxylin (DAKO, Glostrup, Denmark). They were subsequently dehydrated, mounted and observed. Staining was evaluated using a double-blinded method by a neuropathologist and a separate investigator. Sections were classified as follows: -, negative; + , focal and weak immunoreactivity; ++ , diffuse and weak or focal and intense immunoreactivity; and +++ , diffuse and intense immunoreactivity. Data were analyzed 
using SPSS 11.5 (SPSS, Inc., Chicago, IL, USA). Comparisons of high expression rates were performed using a $\chi^{2}$ test. The use of human tissue samples followed internationally recognized guidelines (http://medicalethics.cma.org.cn/), as well as local and national regulations. Research that involved human tissue followed international and national regulations. The present study was approved by the ethics committee of the Second Artillery General Hospital of the People's Liberation Army, Beijing, China.

Cell line and transfection. U87MG human glioblastoma cells were obtained from MD Anderson Cancer Center (Houston, TX, USA). Cells were cultured in RPMI-1640 supplemented with $10 \%$ fetal calf serum (Gibco Life Technologies, Carlsbad, CA, USA). Cells were maintained in a humidified atmosphere containing $5 \% \mathrm{CO}_{2}$ at $37^{\circ} \mathrm{C}$. GK5-expressing plasmids and empty vectors (pcDNA3.1) were purchased from the RNAi Core Facility Platform (Academia Sinica, Taiwan, China). Pre-miR-135b and control-miR (50-nM) were purchased from Ambion Life Technologies (Carlsbad, CA, USA) and used for transfection. Cells were cultured in serum-free medium without antibiotics at $60 \%$ confluence for $24 \mathrm{~h}$, and then transfected using transfection reagent (Lipofectamine $2000^{\circledR}$, Invitrogen Life Technologies, Carlsbad, CA, USA), according to the manufacturer's instructions. Following incubation for $6 \mathrm{~h}$, the medium was removed and replaced with a different culture medium for $48 \mathrm{~h}$.

Western blot analysis. Western blot analysis was performed as described in a previous study (13). Following incubation with rabbit anti-GK5 antibodies overnight at $4{ }^{\circ} \mathrm{C}$, IRDyeTM-800-conjugated anti-rabbit secondary antibodies (cat. no. AF881756; Li-COR, Lincoln, NE, USA) were added for $30 \mathrm{~min}$ at room temperature. Specific proteins were visualized using an Odyssey ${ }^{\mathrm{TM}}$ Infrared Imaging system (Gene Company, Ltd., Hong Kong, China).

Cell cycle analysis. U87MG glioblastoma cells $\left(8.0 \times 10^{5}\right.$ cells $)$ were seeded into a $100 \mathrm{~mm}$ culture plate and incubated overnight. Cells were transfected with plasmids for $24 \mathrm{~h}$, washed twice with $\mathrm{NaCl} / \mathrm{Pi}$ buffer, containing $0.5 \%$ Triton X-100, $1.0 \mathrm{mM}$ phenylmethylsulfonyl fluoride, $10 \mathrm{pg} / \mathrm{ml}$ soybean trypsin inhibitor, $10 \mathrm{pg} / \mathrm{ml}$ aprotinin and $1.0 \mathrm{mM}$ EGTA, and then centrifuged at $200 \mathrm{x}$ g at room temperature for $15 \mathrm{~min}$. Pellets were resuspended in $1 \mathrm{ml}$ cold $\mathrm{NaCl} / \mathrm{Pi}$ and fixed in $70 \%$ ethanol for $<12 \mathrm{~h}$ at $4^{\circ} \mathrm{C}$. Fixed cells were incubated with $100 \mu \mathrm{l}$ DNase-free RNase A $(200 \mu \mathrm{g} / \mathrm{ml})$ for $30 \mathrm{~min}$ at $37^{\circ} \mathrm{C}$ and then $1 \mathrm{mg} / \mathrm{ml}$ propidium iodide was added. Stained cells were analyzed using a fluorescence-activated cell sorter (BD Accuri C6; BD Biosciences, Ann Arbor, MI, USA). The percentages of cells in G0, G1, S and G2/M cell cycles phases were determined using Cell Quest Pro version 5.2.1software (FlowJo, Ashland, OR, USA).

Immunofluorescence staining. Following transfection, the cells were fixed in $4 \%$ paraformaldehyde for $15 \mathrm{~min}$, and then blocked using goat serum blocking solution (Gibco Life Technology, Grand Island, NY, USA) for $20 \mathrm{~min}$ at room temperature. Rabbit antibody against GK5 (1:200 dilution; Abcam) was added and the mixtures were incubated in a humid chamber overnight. Following three wash phases using $\mathrm{NaCl} / \mathrm{Pi}$ buffer, cells were incubated using the appropriate secondary antibodies (fluorescein isothiocyanate-conjugated goat anti-rabbit immunoglobulin G; Santa Cruz Biotechnology, Inc., Dallas, TX, USA) for $30 \mathrm{~min}$ at $37^{\circ} \mathrm{C}$. Samples were then observed under a laser scanning confocal microscope (LSM-GB200; Olympus, Tokyo, Japan). 4'6-Diamidino-2-phenylindole (Boehringer, Mannheim, Germany) staining was used for nuclei visualization.

Reverse transcription-polymerase chain reaction (RT-PCR). Total RNA was isolated from cells using TRIzol ${ }^{\circledR}$ reagent (Invitrogen Life Technologies). cDNA was synthesized from $1 \mu \mathrm{g}$ total RNA using a $20 \mu \mathrm{l}$ RT system followed by PCR amplification in a 50- $\mu$ l PCR system performed using an RT-PCR kit (Promega Corporation, Madison, WI, USA). The reference gene glyceraldehyde-3-phosphate dehydrogenase (GAPDH) was used as an RNA loading control. The PCR primer sequences were as follows: Forward: 5'-GTGCTGTCCGCGGACAAGAAC-3' and reverse: 5'-TGTGAACAATAGCTCTCATC-3' for GK5; and forward: 5'-TGTCATCAACGGGAAGCCCA-3' and reverse: 5'-TTGTC ATGGATGACCTTGGC-3' for GAPDH. The thermal cycle profile was as follows: denaturation for $30 \mathrm{sec}$ at $95^{\circ} \mathrm{C}$, annealing for $45 \mathrm{sec}$ at $58^{\circ} \mathrm{C}$, depending on the primers used, and extension for $45 \mathrm{sec}$ at $72^{\circ} \mathrm{C}$.

PCRs were conducted according to the manufacturer's instructions (Promega, Madison, WI, USA) and PCR products were analyzed using agarose gel electrophoresis. PCR products were visualized on $2 \%$ agarose gels stained with ethidium bromide under UV transillumination and densities of the bands were determined using a computerized image analysis system (Alpha Innotech, San Leandro, CA, USA). The area of each band was calculated as the integrated density value (IDV). Mean values were calculated from three separate experiments. The IDV ratio of GK5 to GAPDH was calculated for each sample.

RT-PCR for miRNAs. The total RNA from cultured cells, with efficient recovery of small RNAs, was isolated using the mirVana miRNA Isolation kit (cat. no. AM1567; Ambion, Austin, TX, USA). Detection of the mature form of miRNAs was performed using the mirVana qRT-PCR miRNA Detection kit (cat. no. AM1558; Ambion), according to the manufacturer's instructions. The U6 small nuclear RNA was used as an internal control.

3-(4, 5-dimethylthiazol-2-yl)-2, 5-diphenyltetrazolium (MTT) assay. Cell proliferation was measured using an MTT assay (Sigma-Aldrich, St. Louis, MO, USA), according to a previously described method (13). Absorbance was directly proportional to the number of surviving cells.

Luciferase reporter assay. The 3'-UTR of human GK 5 mRNA was cloned in between the transcription complex subunit 1 and Xba 1 sites of pRL-TK (Promega Corporation, Madison, WI, USA) using a PCR-generated fragment. Site-directed mutagenesis of the miR-135b target-site in the GK5-3'-UTR was conducted using a Quik change-mutagenesis kit (Agilent 
Table I. GK5 expression in human glioma.

\begin{tabular}{lccc}
\hline Grade & $\mathrm{n}$ & $-/+$ & GK5 ++ (+++) \\
\hline I & 116 & 14 & $102(88 \%)$ \\
IV & 122 & 89 & $33(27 \%)$ \\
\hline
\end{tabular}

GK5, glycerol kinase 5 .

Technologies, Inc., Santa Clara, CA, USA) and GK5-wild type-luciferase (GK5-WT-luc) as a template. In order to conduct the reporter assays, U87MG cells were transiently transfected with WT or mutant reporter plasmid and the appropriate miR, using Lipofectamine 2000. Reporter assays were performed $36 \mathrm{~h}$ post-transfection using a dual-luciferase assay-system (Promega Corporation), they were standardized for transfection efficiency using cotransfected Renilla-luciferase.

Bioinformatics. The analysis of potential microRNA target sites was performed using four commonly used prediction algorithms: miRanda (http://www.microrna.org/), TargetScan (http://www.targetscan.org), miRDB (http://mirdb.org/ miRDB/) and PicTar (http://pictar.bio.nyu.edu).

Statistical analysis. Data are expressed as the mean \pm standard error with three independent experiments and analyzed using Student's t-test. $\mathrm{P}<0.05$ was considered to indicate a statistically significant difference.

\section{Results}

GK5 as a tumor suppressor gene in glioblastoma. In order to measure GK5 expression in glioblastoma, western blotting was conducted in 10 pairs of glioblastoma and adjacent healthy tissue samples. GK5 expression was lower in glioblastoma tissues compared with healthy tissues (Fig. 1A). In a previous study, the invasive capability of glioblastoma was found to be closely associated with its pathological grade (14). In the present study, positive staining for GK5 was observed in the cytoplasm of glioblastoma cells (Fig. 1B). GK5 protein expression levels in glioma grades I and IV were 88 and 27\%, respectively (Table I). Statistical analysis demonstrated that the GK5 expression levels were lower in glioma grade IV samples than in grade I samples $(\mathrm{P}<0.001)$. U87MG cells were transfected with GK5-expressing plasmids. Following transfection, GK5 protein expression was detected using western blot analysis. Results demonstrated that GK5 plasmids may induce GK5 protein expression in U87MG cells (Fig. 1C). Furthermore, cell cycle stages of U87MG cells were analyzed using fluorescence-activated cell sorting (FACS) analysis. The results demonstrated that GK5 overexpression significantly inhibited U87MG cell proliferation compared with control cells (Fig. 1C). Fewer GK5 transfected U87MG cells were observed in the S and G2/M phases compared with control cells (Fig. 1D). The results of the present study suggest that GK5 may be a tumor suppressor gene in glioblastoma.
A

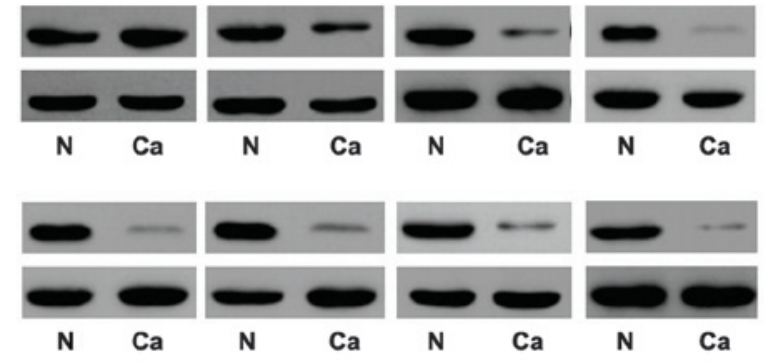

B
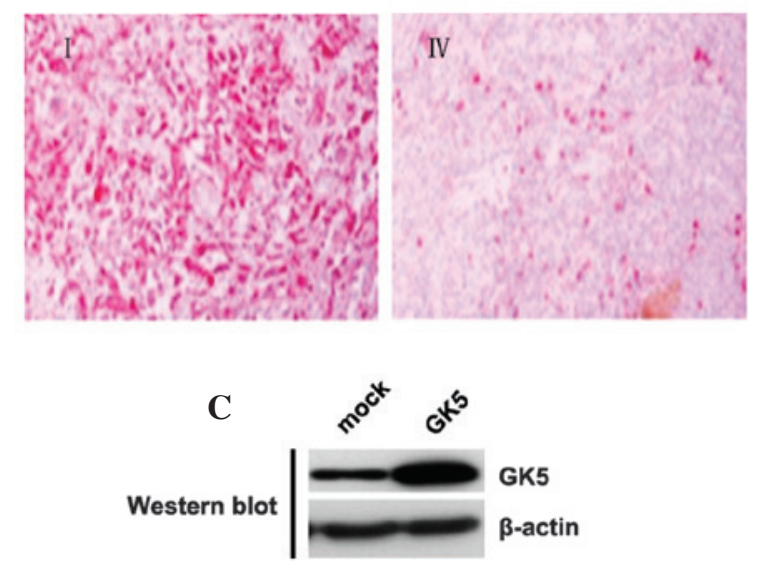

D
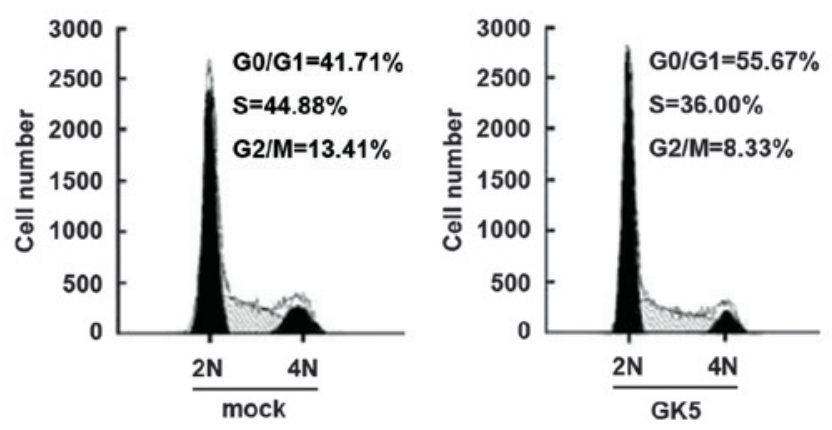

Figure 1. GK5 is a tumor suppressor gene in glioblastoma. (A) Western blot analysis for GK5 protein expression in 8 pairs of glioblastoma and adjacent healthy tissue samples. $\beta$-actin was used as a control. (B) Representative tissue sections of world health organization grade I and IV glioblastoma samples. (C) Western blot analysis for GK5 in U87MG cells transfected with GK5-expressing plasmids or control vectors, $n=3$. (D) FACS analysis for U87MG cells transfected with GK5-expressing plasmids or control vectors. $\mathrm{n}=3$. GK5, glycerol kinase 5; FACS, fluorescence-activated cell sorting; $\mathrm{Ca}$, cancerous.

GK5 is a target of miR-135b in U87MG glioblastoma cells. miRs are small ( 22 nucleotides) non-coding RNAs and they negatively regulate protein-coding gene expression by targeting mRNA degradation or inhibiting translation (15-17). It is hypothesized that GK5 expression is downregulated in glioblastoma in response to the overexpression of particular types of miRNAs. In order to investigate this hypothesis, 3 prediction algorithms were used: miRanda (http://www.microrna.org/),TargetScan(http://www.targetscan.org) and $\mathrm{miRDB}$ (http://mirdb.org/miRDB/) in order to analyze the 3'UTR of NUAK1. According to the results of each prediction 
A

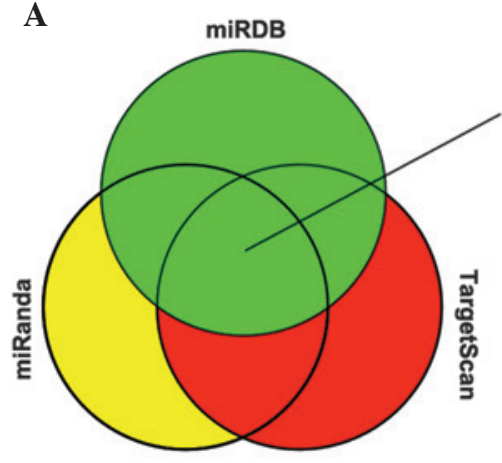

B

miR-135a

miR-135b

miR-145
D

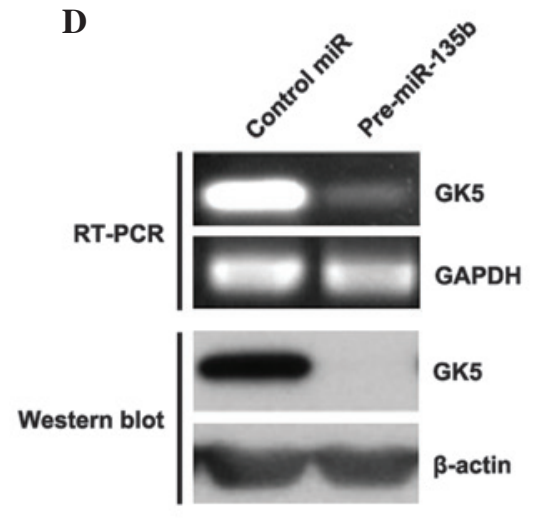

$\mathbf{E}$
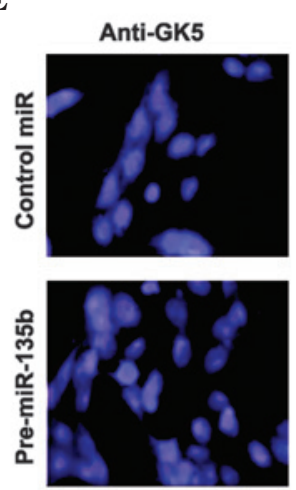

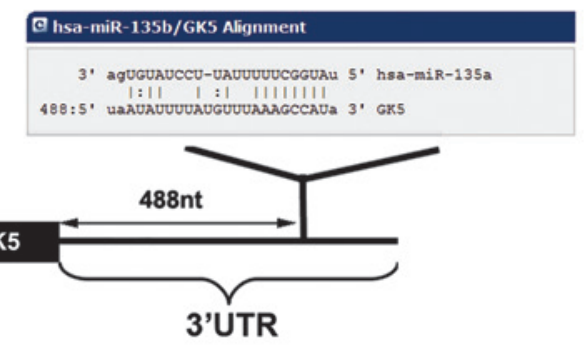

C

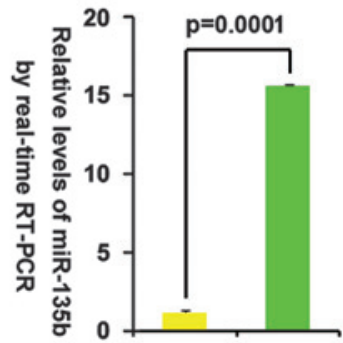

F

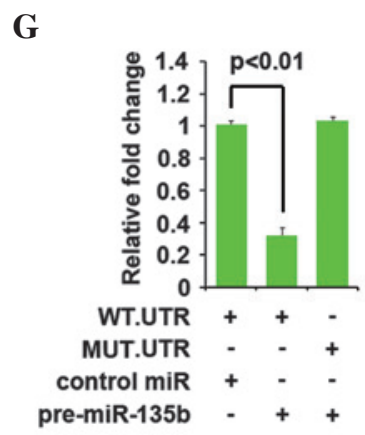

\author{
3' AguguauccuuauUuUucgguaU 5' hsa-miR-135b \\ IIIIIII \\ 5' UAAUAUUUUAUGUUUAAAGCCAUA 3' GK5-WT \\ 5' UaAUAUUUUAUGUUUAAAgGgCUA 3' GK5-MUT
}
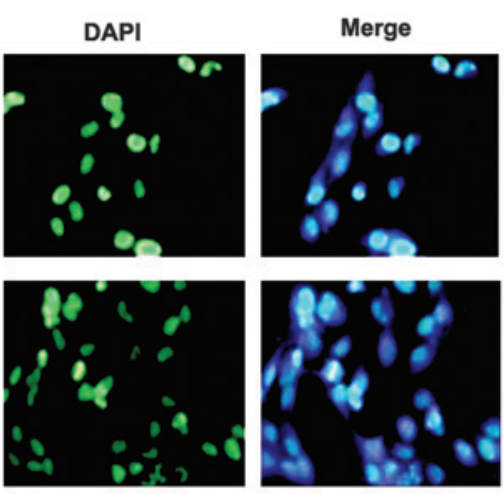

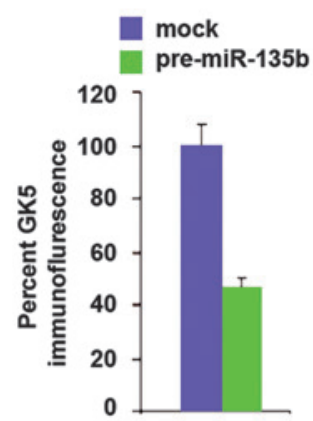

Figure 2. GK5 is a target of miR-135b in U87MG glioblastoma cells. (A) miRanda, TargetScan and miRDB predicted that miR-135b targets the 3'UTR of GK5. (B) Predicted target sites of miR-135b according to miRanda, TargetScan and miRDB databases. (C) RT-PCR for miR-135b in U87MG cells, transfected with control miR or pre-miR-135b. U6 was used as a control, $\mathrm{n}=3$. RT-PCR for GK5 mRNA in U87MG cells transfected with control miR or pre-miR-135b. GAPDH was used as a control, $n=3$. (D) Western blot analysis for GK5 protein expression in U87MG cells transfected with control miR or pre-miR-135b. $\beta$-actin was used as a control, $n=3$. (E) Representative images of immunostained U87MG cells transfected with pre-miR-135b or control miR. Immunofluorescent staining of one representative experiment (left; x100). Mean fluorescence intensities of three independent experiments (right). (F) GK5-3'-UTR containing reporter constructs. MUT, contains a 3-base-mutation at the miR-135b-target region, which suppresses its binding capability. (G) Reporter assay, with cotransfection of $500 \mathrm{ng}$ WT-or MUT-reporter and $50 \mathrm{nM}$ control-miR, or pre-miR-135b as indicated. Following transfection ( $36 \mathrm{~h}$ ), U87MG cells were harvested in order to conduct a luciferase reporter assay, $\mathrm{n}=3$. RT-PCR, reverse transcription-polymerase chain reaction; miR, microRNA; UTR, untranslated region; nt, nucleotide; DAPI, 4',6-diamidino-2-phenylindole; GK5, glycerol kinase 5; WT, wild type.

algorithm miR-135a, miR-135b and miR-145 target the 3'UTR of GK5 (Fig. 2A). It has been reported that miR-135b is upregulated in glioblastoma samples compared with healthy samples (6). Therefore, the present study hypothesized that GK5 downregulation in glioblastoma is a result of miR-135b upregulation. The predicted target sites of miR-135b are summarized in Fig. 2B.

In order to investigate whether miR-135b inhibits GK5 expression, U87MG cells were transfected with pre-miR-135b and RT-PCR was performed. The results demonstrated that transfection with pre-miR-135b significantly increased miR-135b expression in U87MG cells compared with control cells (Fig. 2C). RT-PCR and western blot analysis were then conducted, in order to detect GK5 expression. Results suggested that miR-135b overexpression induced GK5 mRNA and protein downregulation in U87MG cells, compared with control cells (Fig. 2D). Immunofluorescent staining was then performed. Taken together with the data above, the results of immuno- 
A

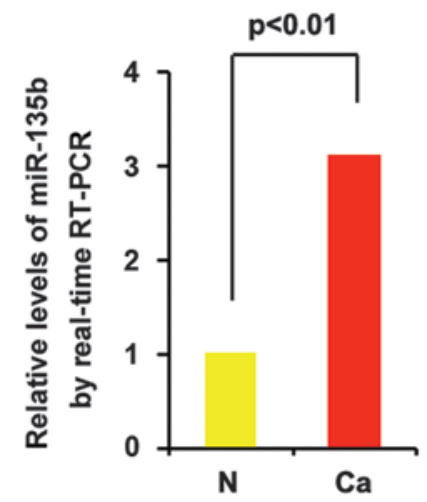

C

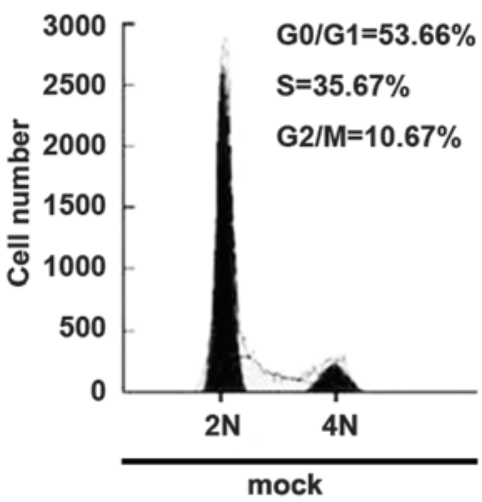

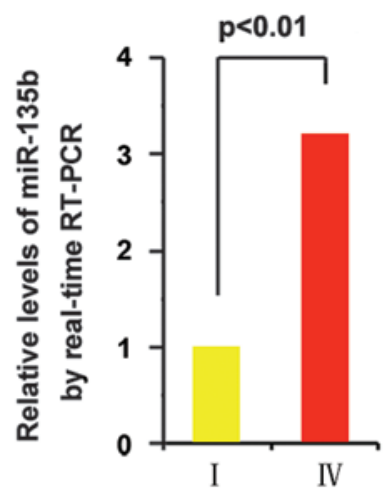

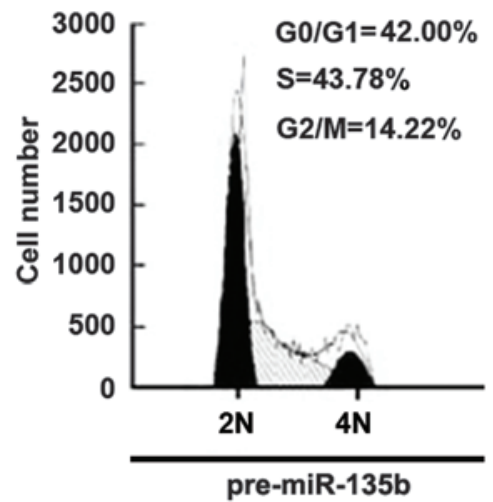

D

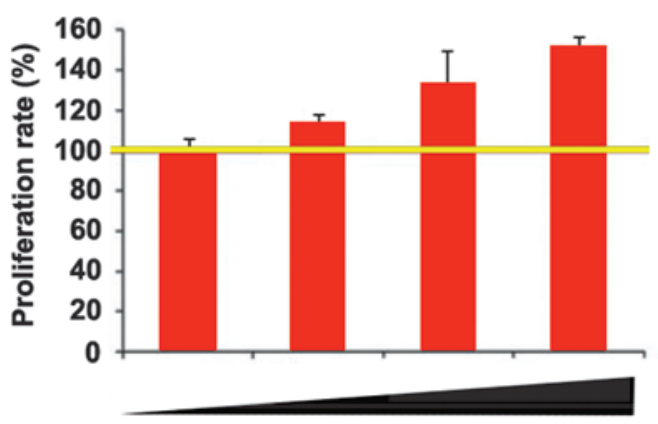

Figure 3. miR-135b is an oncogene in glioblastoma. (A) RT-PCR for miR-135b in 55 pairs of glioblastoma and adjacent healthy tissues. U6 was used as a control. n=55. (B) RT-PCR for miR-135b in 67 glioma tissues (WHO grade I) and glioblastoma tissues (WHO grade IV). U6 was used as a control. n=55. (C) Fluorescence-activated cell sorting analysis for pre-miR-135b or control miR-transfected U87MG cells, n=3. (D) MTT for U87MG cells transfected with pre-miR-135b or control miR (mock), $\mathrm{n}=3$. miR, microRNA; RT-PCR, reverse transcription-polymerase chain reaction; WHO, world health organization; MTT, 3-(4, 5-dimethylthiazol-2-yl)-2, 5-diphenyltetrazolium; Ca, cancerous.

fluorescent staining suggested that pre-miR-135b significantly downregulated GK5 expression in U87MG cells (Fig. 2E).

In order to demonstrate the regulation of GK5 by miR-135b, luciferase reporters were developed using the wild-type targeting sequences (GK5-WT-luc) and the mutated GK5 3'UTRs (GK5-MUT-luc; Fig. 2F). In order to detect whether miR-135b targets the 3'UTR of GK5, a luciferase assay was performed. The results suggested that pre-miR-135b inhibited GK5-WT-luc plasmids but not GK5-MUT-luc plasmids (Fig. 2G). Overall, results of the present study suggested that miR-96 negatively regulates expression of the protein-coding gene GK5, by targeting its 3'UTR. GK5 downregulation is associated with miR-135b expression in glioblastoma.

miR-135b as an oncogene in glioblastoma. It has been reported that $\mathrm{miR}-135 \mathrm{~b}$ expression is upregulated in glioma compared with adjacent healthy tissue samples (6). The present results were consistent with those of a previous study (6) and demonstrated that miR-135 expression is greater in glioblastoma tissues compared with healthy tissues (Fig. 3A). RT-PCR was conducted in order to detect differences in miR-135b expression levels between glioma (WHO grade I) and glioblastoma (WHO grade IV). The 
results demonstrated that miR-135b expression is higher in glioblastoma (WHO grade IV) than in glioma samples (WHO grade I; Fig. 3B). In order to investigate miR-135b expression patterns in vitro, FACS analyses were performed in order to detect cell cycle distribution in pre-miR-135b-transfected U87MG cells. FACS analysis suggested that more $S$ phase cells were observed among pre-miR-135b-cells compared with control cells (Fig. 3C). In order to further confirm whether pre-miR-135b affects U87MG cell proliferation an MTT assay was performed. The results suggested that proliferation rate was increased in pre-miR-135b-transfected cells in a dose-dependent manner (Fig. 3D).

\section{Discussion}

To the best of our knowledge, there are limited studies on the involvement of GK5 in cancer and those focussing on glioblastoma are particularly rare. In the present study, GK5 expression was observed to be greater in glioblastoma tissues than in healthy tissues. In addition, GK5 protein expression rates in grades I and IV tissues were 88 and $27 \%$, respectively (Table I). Therefore, results of the present study implied that GK5 expression was associated with glioma pathological grades. Statistical analysis suggested that the GK5 expression level was lower in grade I vs. IV glioma samples $(\mathrm{P}<0.001)$. Furthermore, the invasive capability of glioblastoma was found to be closely associated with its WHO pathological grade (14). Although migration and invasion assays were not conducted in the present study, it is hypothesized that GK5 expression is associated with migration and invasion in glioblastoma cells in vitro. In accordance with the results from patient tissue samples in vitro, GK5 expression was shown to significantly suppress cell proliferation in the present study.

The involvement of miRs in phenotypic modulation of human glioma has been reported in a previous study. For example, miR-21 knockdown disrupts glioma growth in vivo and exhibits synergistic cytotoxicity with neural precursor cell sTRAIL in human glioma (3). miR-34a is tumor suppressor in brain tumors and glioma stem cells (4) and miR-181a sensitizes U87MG human malignant glioma cells to radiation by targeting Bcl-2 (5). miR-135b expression is upregulated in glioma samples compared with healthy samples (6). In accordance with previous studies, miR-135b expression was upregulated in glioblastoma tissues, compared with adjacent healthy tissues. Furthermore, miR-135b expression was upregulated in glioblastoma WHO IV grade, compared with glioblastoma classified as WHO I grade. The present study suggested that miR-135b regulates GK5 and functions as an oncogene. It would be beneficial to examine the involvement of an miR-135b-knockout in nude mice. This may demonstrate the role of miR-125b in vivo.

Results of miR-135b and GK5 regulation in glioblastoma in the present study provide potential clinical implications. GK5 may be an antitumor suppressor that inhibits glial cell proliferation and evidence suggests that it is associated with glioblastoma cell invasion. Pharmacological restoration of GK5 may represent a novel therapeutic strategy for patients with glioma. miR-135b is associated with the pathogenesis and progression of glioblastoma. Therefore, miR-135b may also represent a potential therapeutic strategy for patients with glioblastoma. However, further research is required in order to better understand the involvement of miR-135b and GK5 in glioblastoma.

\section{References}

1. Miwa K, Matsuo M, Ogawa S, et al: Hypofractionated high dose irradiation with positron emission tomography data for the treatment of glioblastoma multiforme. Biomed Res Int 2014: 407026, 2014.

2. Baek D, Villén J, Shin C, Camargo FD, Gygi SP and Bartel DP: The impact of microRNAs on protein output. Nature 455: 64-71, 2008.

3. Corsten MF, Miranda R, Kasmieh R, Krichevsky AM, Weissleder R and Shah K: MicroRNA-21 knockdown disrupts glioma growth in vivo and displays synergistic cytotoxicity with neural precursor cell delivered S-TRAIL in human gliomas. Cancer Res 67: 8994-9000, 2007.

4. Guessous F, Zhang Y, Kofman A, Catania A, Li Y, Schiff D, Purow B and Abounader R: MicroRNA-34a is tumor suppressive in brain tumors and glioma stem cells. Cell Cycle 9: 1031-1036, 2010.

5. Chen G, Zhu W, Shi D, Lv L, Zhang C, Liu P and Hu W: MicroRNA-181a sensitizes human malignant glioma U87MG cells to radiation by targeting Bcl-2. Oncol Rep 23: 997-1003, 2010.

6. Lavon I, Zrihan D, Granit A, Einstein O, Fainstein N, Cohen MA, Cohen MA, Zelikovitch B, Shoshan Y, Spektor S, et al: Gliomas display a microRNA expression profile reminiscent of neural precursor cells. Neuro Oncol 12: 422-433, 2010.

7. Arigoni M, Barutello G, Riccardo F, Ercole E, Cantarella D, Orso F, Conti L, Lanzardo S, Taverna D, Merighi I, et al: miR-135b coordinates progression of ErbB2-driven mammary carcinomas through suppression of MID1 and MTCH2. Am J Pathol 182: 2058-2070, 2013.

8. Lin CW, Chang YL, Chang YC, Lin JC, Chen CC, Pan SH, Wu CT, Chen HY, Yang SC, Hong TM, et al: MicroRNA-135b promotes lung cancer metastasis by regulating multiple targets in the Hippo pathway and LZTS1. Nat Commun 4: 1877,2013

9. Zhang L, Sun ZJ, Bian Y and Kulkarni AB: MicroRNA-135b acts as a tumor promoter by targeting the hypoxia-inducible factor pathway in genetically defined mouse model of head and neck squamous cell carcinoma. Cancer Lett 331: 230-238, 2013.

10. Matsuyama H, Suzuki HI, Nishimori H, Noguchi M, Yao T, Komatsu N, Mano H, Sugimoto K and Miyazono K: miR-135b mediates NPM-ALK-driven oncogenicity and renders IL-17-producing immunophenotype to anaplastic large cell lymphoma. Blood 118: 6881-6892, 2011

11. Khatri R and Subramanian S: MicroRNA-135b and its circuitry networks as potential therapeutic targets in colon cancer. Front Oncol 3: 268, 2013.

12. Louis D, Ohgaki H, Wiestler OD, et al: The 2007 WHO classification of tumours of the central nervous system. Acta Neuropathol 114: 97-109, 2007.

13. Liao XH,Lu DL, Wang N,Liu LY, Wang Y,Li YQ, Yan TB, Sun XG, $\mathrm{Hu} \mathrm{P}$ and Zhang TC: Estrogen receptor $\alpha$ mediates proliferation of breast cancer MCF-7 cells via a p21/PCNA/E2F1-dependent pathway. FEBS J 281: 927-942, 2014.

14. Ohgaki $\mathrm{H}$ and Kleihues P: Genetic pathways to primary and secondary glioblastoma. Am J Pathol 170: 1445-1453, 2007.

15. Lee RC, Feinbaum RL and Ambros V: The C. elegans heterochronic gene lin-4 encodes small RNAs with antisense complementarity to lin-14. Cell 75: 843-854, 1993.

16. Pasquinelli AE, Reinhart BJ, Slack F, Martindale MQ, Kuroda MI, Maller B, Hayward DC, Ball EE, Degnan B, Müller P, et al: Conservation of the sequence and temporal expression of let-7 heterochronic regulatory RNA. Nature 408: 86-89, 2000.

17. Reinhart BJ, Slack FJ, Basson M, Pasquinelli AE, Bettinger JC, Rougvie AE, Horvitz HR and Ruvkun G: The 21-nucleotide let-7 RNA regulates developmental timing in Caenorhabditis elegans. Nature 403: 901-906, 2000 